\title{
Partially Observed Markov Decision Process Multiarmed Bandits-Structural Results
}

\author{
Vikram Krishnamurthy \\ Department of Electrical and Computer Engineering, University of British Columbia, Vancouver, \\ British Columbia V6T 1Z4, Canada, vikramk@ece.ubc.ca, http://www.ece.ubc.ca/ vikramk \\ Bo Wahlberg \\ Automatic Control and ACCESS, School of Electrical Engineering, KTH, SE-100 44 Stockholm, Sweden, \\ bo.wahlberg@ee.kth.se
}

\begin{abstract}
This paper considers multiarmed bandit problems involving partially observed Markov decision processes (POMDPs). We show how the Gittins index for the optimal scheduling policy can be computed by a value iteration algorithm on each process, thereby considerably simplifying the computational cost. A suboptimal value iteration algorithm based on Lovejoy's approximation is presented. We then show that for the case of totally positive of order 2 (TP2) transition probability matrices and monotone likelihood ratio (MLR) ordered observation probabilities, the Gittins index is MLR increasing in the information state. Algorithms that exploit this structure are then presented.

Key words: multiarmed bandits; partially observed Markov decision process; monotone policies; likelihood ratio ordering; opportunistic scheduling; stochastic approximation algorithm MSC2000 subject classification: Primary: 90Bxx; secondary: 93Exx

OR/MS subject classification: Primary: dynamic programming/optimal control; secondary: stochastic scheduling

History: Received July 24, 2008; revised December 3, 2008. Published online in Articles in Advance April 10, 2009.
\end{abstract}

1. Introduction. The multiarmed bandit problem is an example of a dynamic stochastic scheduling problem for optimizing in a sequential manner the allocation effort between a number of competing projects. Numerous applications of this finite-state Markov chain multiarmed bandit problems appear in the operations research and stochastic control literature, see Gittins [5] and Whittle [20] for examples in job scheduling and resource allocation for manufacturing systems. The multiarmed bandit problem structure implies that the optimal policy can be found by a so-called Gittins [5] index rule. This implies that the multiproject optimization problem is simplified to a finite number of single project optimization problems. Several algorithms have been proposed to solve this fully observed finite-state bandit problem (Kumar and Varaiya [9], Bertsimas and Nino-Mora [1]).

This paper considers bandit problems where the underlying finite-state Markov chain is not directly observedinstead the observations (which are assumed to belong to a finite set) are a probabilistic function of the unobserved finite-state Markov chain. Such problems are known as partially observed Markov decision process (POMDP) multiarmed bandits and are also called Hidden Markov Model (HMM) multiarmed bandits. POMDPs have recently received much attention in the area of artificial intelligence for autonomous robot navigation-see Cassandra [2] for a nice web-based tutorial. They have also been used for optimal observer trajectory planning in bearings only target tracking - we refer the reader to Le Cadre and Trémois [11] for an excellent exposition.

In general, solving POMDPs are computationally intractable apart from examples with small state and action spaces. In complexity theory (Papadimitriou [15]), they are known as PSPACE hard problems requiring exponential memory and computation. For partially observed scheduling problems involving several tens or hundreds of processes, the POMDP has an underlying state space that is exponential in the number of processes-which is prohibitively expensive to solve. However, for a multiarmed bandit problem, the optimal policy can be found by a so-called Gittins index rule (Gittins [5], Ross [17]). This means that at each time instant, the optimal action is to choose the process with the highest Gittins index, where the Gittins index of each process is a function of the state of that process only. Therefore the problem essentially decouples into solving individual POMDPs for each process. There are numerous applications of multiarmed bandit problems (Gittins [5], Whittle [20]).

1.1. Main results. Given the multiarmed bandit POMDP formulation and the indexable nature of the optimal policy, the main issue is how to compute the Gittins index for the individual processes. While there are several algorithms available for computing the Gittins indices for fully observed Markov decision process bandit problems (Kumar and Varaiya [9]), our POMDP bandit problem is more difficult because underlying finite-state Markov chain is not directly observed-instead the observations are a probabilistic function of the unobserved finite-state Markov chain. The main contribution of this paper is to use two classes of methods to compute the Gittins index for POMDP multiarmed bandits. 
1.1.1. Value iteration algorithms for computing Gittins index. In $\S 3$, we present finite-dimensional value iteration algorithm for computing the Gittins index. We show that by introducing the retirement formulation (Gittins [5]) of the multiarmed bandit problem option, a finite-dimensional value iteration algorithm can be derived for computing the Gittins index of a POMDP bandit. The key idea is to extend the state vector to include retirement information. The main outcome of $\S 3$ is that the Gittins index $\gamma(x)$ at information state $x$ is a rational function of $x$ (ratio of two linear polynomials in $x$ ); see Theorem 3.1.

In light of the above characterization of the Gittins index, it can be computed numerically by any number of methods that have been developed for solving POMDPs (see §3.2). We also present a suboptimal algorithm for computing the Gittins index using Lovejoy's [13] value iteration approximation.

1.1.2. Monotone Gittins index and linear approximation. The value iteration-based methods mentioned above for computing the Gittins index can become computationally intractable for large state and observation spaces. In $\S 4$, we show that under reasonable model assumptions (monotone increasing rewards, totally positive of order 2 (TP2) transition probability matrix and observation probability matrix), the Gittins index in monotone in the information state (in terms of the monotone likelihood ratio (MLR) order). Thus the optimal policy is opportunistic in that if the information states of the $P$ projects are MLR comparable, then the optimal policy is to pick the project with the largest MLR information state. This requires negligible computational cost to implement. Of course, in general, the information states of the processes may not be MLR comparable (because the MLR ordering is a partial order). Therefore we propose a linear approximation to the Gittins index and give a necessary and sufficient condition for it to be MLR increasing. The resulting best linear approximation to the Gittins index yields a computationally simple policy that coincides with the optimal policy when the states are MLR comparable. We then give a stochastic approximation algorithm to estimate the best linear approximation of the Gittins index. Finally, we present another approximation where non-MLR comparable information states are projected onto the nearest MLR comparable state. This turns out to be a convex optimization problem and can be solved efficiently.

In $\S 5$, numerical examples are presented. The performance of the suboptimal algorithm for computing the Gittins index based on Lovejoy's approximation is also illustrated.

2. The POMDP multiarmed bandit problem. Consider $P$ independent projects $p=1, \ldots, P$. Assume each project $p$ has a finite number of states $\mathcal{N}_{p}$. Let $s_{k}^{(p)}$ denote the state of project $p$ at discrete time $k=$ $0,1, \ldots$. At each time instant $k$, only one of these projects can be worked on. If project $p$ is worked on at time $k$, an instantaneous reward $\beta^{k} R\left(s_{k}^{(p)}, p\right)$ is accrued $\left(R\left(s_{k}, p\right) \geq 0\right.$ is assumed finite). Here, $0 \leq \beta<1$ denotes the discount factor; the state $s_{k}^{(p)}$ evolves according to an $\mathcal{N}_{p}$-state homogeneous Markov chain with transition probability matrix

$$
A^{(p)}=\left(a_{i j}^{(p)}\right)_{i, j \in N_{p}}, \quad \text { where }=a_{i j}^{(p)}=P\left(s_{k+1}^{(p)}=j \mid s_{k}^{(p)}=i\right) \text { if project } p \text { is worked on at time } k .
$$

The states of all the other $(P-1)$ idle projects are unaffected, i.e., $s_{k+1}^{(p)}=s_{k}^{(p)}$, if project $p$ is idle at time $k$. All projects are initialized with $s_{0}^{(p)} \sim x_{0}^{(p)}$, where $x_{0}^{(p)}$ are specified initial distributions for $p=1, \ldots, P$.

The state of the active project $p$ is indirectly observed via noisy measurements (observations) $y_{k+1}^{(p)}$ of the active project state $s_{k+1}^{(p)}$. Assume that these observations $y_{k+1}^{(p)}$ belong to a finite set $\mu_{p}$ indexed by $m^{(p)}=1, \ldots, M_{p}$. Let $B^{(p)}=\left(b_{i m}^{(p)}\right)_{i \in \mathcal{N}_{p}, m \in \mu_{p}}$ denote the observation probability (symbol probability) matrix of the (HMM), where each element $b_{i m}^{(p)} \triangleq P\left(y_{k+1}^{(p)}=m \mid s_{k+1}^{(p)}=i, u_{k}=p\right)$.

Let $u_{k} \in\{1, \ldots, P\}$ denote which project is worked on at time $k$. Consequently, $s_{k+1}^{\left(u_{k}\right)}$ denotes the state of the active project at time $k+1$. Denote the observation history at time $k$ as $Y_{k}=\left(y_{1}^{\left(u_{0}\right)}, \ldots, y_{k}^{\left(u_{k-1}\right)}\right)$ and let $U_{k}=\left(u_{0}, \ldots, u_{k}\right)$. Then the project at time $k+1$ is chosen according to $u_{k+1}=\mu\left(Y_{k+1}, U_{k}\right)$, where the policy denoted as $\mu$ belongs to the class of stationary policies $U$. The total expected discounted reward over an infinite-time horizon is given by

$$
J_{\mu}=\mathbf{E}\left[\sum_{k=0}^{\infty} \beta^{k} R\left(s_{k}^{\left(u_{k}\right)}, u_{k}\right)\right], \quad u_{k}=\mu\left(Y_{k}, U_{k-1}\right),
$$

where $\mathbf{E}$ denotes mathematical expectation. The aim is to determine the optimal stationary policy $\mu^{*}=$ $\arg \max _{\mu \in \mathscr{U}} J_{\mu}$, which yields the maximum reward in (2). 
2.1. Information state formulation. The above partially observed multiarmed bandit problem can be re-expressed as a fully observed multiarmed bandit in terms of the information state (Kumar and Varaiya [9]). For each project $p$, denote by $x_{k}^{(p)}$ the information state at time $k$ (Bayesian posterior distribution of $s_{k}^{(p)}$ ) as

$$
x_{k}^{(p)}=\left(x_{k}^{(p)}(i)\right) \quad i=1, \ldots, \mathcal{N}_{p}, \text { where } x_{k}^{(p)}(i) \triangleq P\left(s_{k}^{(p)}=i \mid Y_{k}, U_{k-1}\right) .
$$

The HMM multiarmed bandit problem can be viewed as the following scheduling problem: Consider $P$ parallel HMM state estimation filters, one for each project. The project $p$ is active, an observation $y_{k+1}^{(p)}$ is obtained and the information state $x_{k+1}^{(p)}$ is computed recursively by the HMM state filter (also known as the "forward algorithm" or "Baum's algorithm" Rabiner [16]) according to

$$
\begin{gathered}
x_{k+1}^{(p)}=T^{(p)}\left(x_{k}^{(p)}, y_{k+1}^{(p)}\right) \quad \text { if project } p \text { is worked on at time } k \\
\text { where } T^{(p)}\left(x^{(p)}, y^{(p)}\right) \triangleq \frac{B^{(p)}\left(y^{(p)}\right) A^{(p) \prime} x^{(p)}}{d\left(x^{(p)}, y^{(p)}\right)}, \quad d^{(p)}\left(x^{(p)}, y^{(p)}\right) \triangleq \mathbf{1}_{\mathcal{N}_{p}}^{\prime} B^{(p)}\left(y^{(p)}\right) A^{(p)^{\prime}} x^{(p)} .
\end{gathered}
$$

In (5) if $y^{(p)}=m$, then $B^{(p)}(m)=\operatorname{diag}\left[b_{1 m}^{(p)}, \ldots, b_{\mathcal{N}_{p}, m}^{(p)}\right]$ is the diagonal matrix formed by the $m$ th column of the observation matrix $B^{(p)}$ and $\mathbf{1}_{\mathcal{N}_{p}}$ is an $\mathcal{N}_{p}$-dimensional column vector of ones.

The state estimates of the other $P-1$ projects remain unaffected, i.e.,

$$
x_{k+1}^{(q)}=x_{k}^{(q)} \quad \text { if project } q \text { is not worked on, } q \in\{1, \ldots, P\}, q \neq p .
$$

Let $\mathscr{X}^{(p)}$ denote the state space of information states $x^{(p)}, p \in\{1,2, \ldots, P\}$, which is an $\left(\mathcal{N}_{p}-1\right)$-dimensional simplex:

$$
\mathscr{X}^{(p)}=\left\{x^{(p)} \in \mathbb{R}^{\mathcal{N}_{p}}: \mathbf{1}_{\mathcal{N}_{p}}^{\prime} x^{(p)}=1,0 \leq x^{(p)}(i) \leq 1 \text { for all } i \in\left\{1, \ldots, \mathcal{N}_{p}\right\}\right\}
$$

The process $x_{k}^{(p)}, p=1, \ldots, P$, qualifies as an information state since (as is well-known Kumar and Varaiya [9]) choosing $u_{k+1}=\mu\left(Y_{k+1}, U_{k}\right)$ is equivalent to choosing $u_{k+1}=\mu\left(x_{k+1}^{(1)}, \ldots, x_{k+1}^{(P)}\right)$. Using the smoothing property of conditional expectations, the reward functional (2) can be rewritten in terms of the information state as

$$
J_{\mu}=\mathbf{E}\left[\sum_{k=0}^{\infty} \beta^{k} R^{\prime}\left(u_{k}\right) x_{k}^{\left(u_{k}\right)}\right], \quad u_{k}=\mu\left(x_{k}^{(1)}, \ldots, x_{k}^{(P)}\right),
$$

where $R\left(u_{k}\right)$ denotes the $\mathcal{N}_{u_{k}}$ dimensional reward column vector $\left[R\left(s_{k}^{(p)}=1, u_{k}\right), \ldots, R\left(s_{k}^{(p)}=\mathcal{N}_{u_{k}}, u_{k}\right)\right]^{\prime}$, and $R\left(s_{k}^{(p)}, u_{k}\right)$ is defined as in (2). The aim is to compute the optimal policy $\arg \max _{\mu \in \mathscr{U}} J_{\mu}$.

It is well known (Whittle [20]) that the optimal policy has an indexable rule (Kumar and Varaiya [9], Whittle [20]): for each project $p$, there is a function $\gamma^{(p)}\left(x_{k}^{(p)}\right)$ called the Gittins index, which is only a function of the project $p$ and the information state $x_{k}^{(p)}$, whereby the optimal scheduling policy at time $k$ is to work on the project with the largest Gittins index, i.e., the optimal policy is

$$
\mu^{*}\left(x_{k}^{(1)}, x_{k}^{(2)}, \ldots, x_{k}^{(P)}\right)=\max _{p \in\{1, \ldots, P\}}\left\{\gamma^{(p)}\left(x_{k}^{(p)}\right)\right\}
$$

Thus, computing the Gittins index is a key requirement for solving any multiarmed bandit problem. (For a formal definition of the Gittins index in terms of stopping times, see Kumar and Varaiya [9]. We will work with a more convenient equivalent definition in terms of the parameterized retirement reward $M$ (Gittins [5]).)

The fundamental problem for the POMDP case is that the Gittins index $\gamma^{(p)}\left(x_{k}^{(p)}\right)$ must be evaluated for each $x_{k}^{(p)} \in \mathscr{X}^{(p)}$, where $\mathscr{X}^{(p)}$ is an uncountable set. In contrast, for the standard finite-state Markov multiarmed bandit problem considered extensively in the literature (e.g., Gittins [5]), the Gittins index can be straightforwardly computed. In $\S 3$, we present a finite-dimensional value iteration algorithm for computing the Gittins index.

3. Value iteration algorithm for computing Gittins index. This section presents a value iteration algorithm for computing the Gittins index $\gamma^{(p)}\left(x^{(p)}\right)$ for each project $p \in\{1,2, \ldots, P\}$. As with any dynamic programming formulation, the computation of the Gittins index for each project $p$ is offline, independent of the Gittins indices of the other $P-1$ projects and can be done a priori.

For each project $p$, define $\bar{M}^{(p)}$ and $B$ as

$$
\bar{M}^{(p)} \triangleq \max _{i \in \mathcal{N}_{p}} R\left(s_{k}^{(p)}=i, u_{k}=p\right) /(1-\beta), \quad B=\max _{p \in\{1, \ldots, P\}} \bar{M}^{(p)} .
$$


Let $M^{(p)}$ denote a positive real number in the interval $\left[0, \bar{M}^{(p)}\right]$. To simplify subsequent notation, we omit the superscript $p$ in $M^{(p)}$ and $\bar{M}^{(p)}$. The Gittins index (Gittins [5]) of project $p$ with information state $x^{(p)}$ can be defined as

$$
\gamma^{(p)}\left(x^{(p)}\right) \triangleq \min \left\{M: V^{(p)}\left(x^{(p)}, M\right)=M\right\}
$$

where $V^{(p)}\left(x^{(p)}, M\right)$ satisfies the functional Bellman's recursion

$$
V^{(p)}\left(x^{(p)}, M\right)=\max \left\{R^{\prime}(p) x^{(p)}+\beta \sum_{m=1}^{\mu_{p}} V^{(p)}\left(T^{(p)}\left(x^{(p)}, m\right)\right) d^{(p)}\left(x^{(p)}, m\right), M\right\},
$$

where $M$ denotes the parameterized retirement reward and $T^{(p)}(\cdot), d^{(p)}(\cdot)$ are defined in (5).

The goal of this section is to transform the above recursion in the form of a standard POMDP. This is done in \$3.1. We introduce an elementary coordinate transformation in (16) to achieve this. Because for any finite horizon, the value function of a POMDP is piecewise linear and convex, we then obtain a finite-dimensional characterization of the Gittins index. Theorem 3.1 gives an explicit characterization of the Gittins index. Then in $\S 3.2$, we present value iteration algorithms to compute the Gittins index.

We start with the following result whose proof is straightforward (see Ross [17]).

Lemma 3.1. For information state $x^{(p)} \in \mathscr{L}^{(p)}$, the optimal reward function $V^{(p)}\left(x^{(p)}, M\right)$ has the following properties as a function of $M$.

(i) $V^{(p)}\left(x^{(p)}, M\right) \geq M$ for all $M$. Moreover, $V^{(p)}\left(x^{(p)}, M\right)$ is convex and monotonically nondecreasing, $V^{(p)}\left(x^{(p)}, M\right)$ is constant for $M \leq-B /(1-\beta)$ and $V^{(p)}\left(x^{(p)}, M\right)=M$ for all $M \geq B /(1-\beta)$.

(ii) Therefore $V^{(p)}\left(x^{(p)}, M\right)-M$ is decreasing in $M$.

The $N$ th order approximation of $V^{(p)}\left(x^{(p)}, M\right)$ is obtained as the following value iteration algorithm $k=1, \ldots, N$ :

$$
V_{k+1}^{(p)}\left(x^{(p)}, M\right)=\max \left[R^{\prime}(p) x^{(p)}+\beta \sum_{m=1}^{M_{p}} V_{k}^{(p)}\left(T^{(p)}\left(x^{(p)}, m\right)\right) d^{(p)}\left(x^{(p)}, m\right), M\right] .
$$

Here, $V_{N}\left(x^{(p)}, M\right)$ is the value function of an $N$-horizon dynamic programming recursion. Let $\gamma_{N}^{(p)}\left(x^{(p)}\right)$ denote the approximate Gittins index computed via the value iteration algorithm (12), i.e.,

$$
\gamma_{N}^{(p)}\left(x^{(p)}\right) \triangleq \min \left\{M: V_{N}^{(p)}\left(x^{(p)}, M\right)=M\right\}
$$

It is well known (Lovejoy [13]) that $V^{(p)}\left(x^{(p)}, M\right)$ can be uniformly approximated arbitrarily closely by a finite-horizon value function $V_{N}^{(p)}\left(x^{(p)}, M\right)$ of (12). A straightforward application of this result shows that the finite-horizon Gittins index approximation $\gamma_{N}^{(p)}\left(x^{(p)}\right)$ of (13) can be made arbitrarily accurate by choosing the horizon $N$ sufficiently large. This is summarized in the following corollary.

COROLlary 3.1. The (infinite-horizon) Gittins index $\gamma^{(p)}\left(x^{(p)}\right)$ of state $x^{(p)}$ can be uniformly approximated arbitrarily closely by the near-optimal Gittins index $\gamma_{N}^{(p)}\left(x^{(p)}\right)$ computed according to (13) for the finitehorizon $N$. In particular, for any $\delta>0$, there exists a finite-horizon $\bar{N}$ such that

(i) $\sup _{x^{(p)} \in \mathscr{C}(p)}\left|\gamma_{\bar{N}-1}^{(p)}\left(x^{(p)}\right)-\gamma_{\bar{N}}^{(p)}\left(x^{(p)}\right)\right| \leq \delta$.

(ii) For this $\bar{N}, \sup _{x^{(p)} \in \mathscr{\ell}}(p)\left|\gamma_{\bar{N}-1}^{(p)}\left(x^{(p)}\right)-\gamma^{(p)}\left(x^{(p)}\right)\right| \leq 2 \beta \delta /(1-\beta)$.

3.1. Finite-dimensional characterization of Gittins index. Unfortunately, the value iteration recursion (12) does not directly translate into practical solution methodologies. At each iteration $k$, one needs to compute $V_{k}^{(p)}\left(x^{(p)}, M\right)$ over an uncountable set, since $x^{(p)} \in \mathscr{X}^{(p)}$ and $M \in[0, \bar{M}]$. The main contribution of this section is to construct a finite-dimensional characterization for the value function $V_{k}^{(p)}\left(x^{(p)}, M\right), k=1,2, \ldots, N$, and hence the near-optimal Gittins index $\gamma_{N}^{(p)}\left(x^{(p)}\right)$. We will show that under a different coordinate basis $V_{k}^{(p)}\left(x^{(p)}, M\right)$ is piecewise linear and convex. Then, computing $\gamma_{N}^{(p)}\left(x^{(p)}\right)$ in (13) simply amounts to evaluating $V_{k}^{(p)}\left(x^{(p)}, M\right)$ at the hyperplanes formed by the intersection of the piecewise linear segments.

Define the $\left(\mathcal{N}_{p}+1\right)$-dimensional augmented information state $\bar{x} \in\left\{\left[x^{\prime}, 0\right]^{\prime},\left[\mathbf{0}_{\mathcal{N}_{p}}^{\prime}, 1\right]^{\prime}\right\}$, where $x \in \mathscr{L}^{(p)}$ is as in (3). As described below, $\bar{x}_{k}=\left[\mathbf{0}_{\mathcal{N}_{p}}^{\prime}, 1\right]^{\prime}$ is interpreted as the "retirement" information state. Define an augmented 
observation process $\bar{y}_{k} \in\left\{1, \ldots, \mathcal{M}_{p}+1\right\}$ and the corresponding $\left(\mathcal{N}_{p}+1\right) \times\left(\mathcal{N}_{p}+1\right)$ transition and observation probability matrices as

$$
\begin{aligned}
A_{1}^{(p)}=\left[\begin{array}{ll}
A^{(p)} & \mathbf{0}_{\mathcal{N}_{p}} \\
\mathbf{0}_{\mathcal{N}_{p}}^{\prime} & 1
\end{array}\right], & B_{1}^{(p)}=\left[\begin{array}{ll}
B^{(p)} & \mathbf{0}_{\mathcal{N}_{p}} \\
\mathbf{0}_{\mathcal{N}_{p}}^{\prime} & 1
\end{array}\right], \\
A_{2}^{(p)}=\left[\begin{array}{ll}
\mathbf{0}_{\mathcal{N}_{p} \times N_{p}} & \mathbf{1}_{\mathcal{N}_{p}} \\
\mathbf{0}_{\mathcal{N}_{p}}^{\prime} & 1
\end{array}\right], & B_{2}^{(p)}=I_{\left(\mathcal{N}_{p}+1\right) \times\left(\mathcal{N}_{p}+1\right)}
\end{aligned}
$$

$$
B_{1}^{(p)}(m)=\operatorname{diag}\left(\text { column } m \text { of } B_{1}^{(p)}\right) ; \quad B_{2}^{(p)}(m)=\operatorname{diag}\left(\operatorname{column} m \text { of } B_{2}^{(p)}\right), \quad m \in\left\{1, \ldots, \mu_{p}+1\right\} .
$$

To construct a finite-dimensional representation of $V^{(p)}\left(x^{(p)}, M\right)$, we will present a coordinate transformation under which $V^{(p)}\left(x^{(p)}, M\right)$ is the value function of a standard POMDP, and $\left(x^{(p)}, M\right)$ is an invertible map to the information state of this POMDP. Because $0 \leq M \leq \bar{M}$, define the pseudoinformation state

$$
z \triangleq\left[\begin{array}{c}
M / \bar{M} \\
1-M / \bar{M},
\end{array}\right], \quad 0 \leq M \leq \bar{M} .
$$

Define the information state $\pi$ and the following coordinate transformation:

$$
\begin{gathered}
\pi=z \otimes \bar{x} \\
\bar{A}_{1}^{(p)}=I_{2 \times 2} \otimes A_{1}^{(p)}=\left[\begin{array}{cc}
A_{1}^{(p)} & 0 \\
0 & A_{1}^{(p)}
\end{array}\right], \quad \bar{A}_{2}^{(p)}=I_{2 \times 2} \otimes A_{2}^{(p)}=\left[\begin{array}{cc}
A_{2}^{(p)} & 0 \\
0 & A_{2}^{(p)}
\end{array}\right], \\
\bar{B}_{1}^{(p)}=I_{2 \times 2} \otimes B_{1}^{(p)}, \quad \bar{B}_{2}^{(p)}=I_{2 \times 2} \otimes B_{2}^{(p)} \\
\bar{B}_{1}^{(p)}(m)=\operatorname{diag}\left(\text { column } m \text { of } \bar{B}_{1}^{(p)}\right) ; \quad \bar{B}_{2}^{(p)}(m)=\operatorname{diag}\left(\operatorname{column} m \text { of } \bar{B}_{2}^{(p)}\right), \quad m \in\left\{1, \ldots, M_{p}+1\right\} \\
\bar{R}_{1}(p)=\left[\begin{array}{llll}
R^{\prime}(p) & 0 & R^{\prime}(p) & 0
\end{array}\right]^{\prime}, \quad \bar{R}_{2}(p)=\left[\begin{array}{llll}
\bar{M} \mathbf{1}_{\mathcal{N}_{p}}^{\prime} & 0 & \mathbf{0}_{\mathcal{N}_{p}}^{\prime} & 0
\end{array}\right]^{\prime} .
\end{gathered}
$$

It is easily shown that $\bar{A}_{1}^{(p)}, \bar{A}_{2}^{(p)}$ are transition probability matrices (their rows add to one and each element is positive) and $\bar{B}_{1}^{(p)}(m), \bar{B}_{2}^{(p)}(m)$ are observation probability matrices. Also, the $2\left(\mathcal{N}_{p}+1\right)$-dimensional vector $\pi^{(p)}$ is an information state since it belongs to $\Pi^{(p)}$, where

$$
\Pi^{(p)} \triangleq\left\{\pi: \mathbf{1}_{2\left(\mathcal{N}_{p}+1\right)}^{\prime} \pi^{(p)}=1 \text {, and } \pi^{(p)}(i) \geq 0 i=1,2, \ldots, 2\left(\mathcal{N}_{p}+1\right)\right\} .
$$

Finally, define the control variable $\nu_{k} \in\{1,2\}$ at each time $k$, where $\nu_{k}$ maps $\pi_{k}$ to $\{1,2\}$ at each time $k$. Note $\nu_{k}=1$ means continue and $\nu_{k}=2$ means retire. Define the policy sequence $\nu=\left(\nu_{1}, \ldots, \nu_{k}\right)$. (The policy $\nu$ is used to compute the Gittins index of project $p$. It is not to be confused with the policy $\mu$ defined in $\S 2$, which determines which project to work on.)

Consider now the following POMDP problem: Parameters $\bar{A}_{2}^{(p)}, \bar{B}_{2}^{(p)}, \bar{R}(2)$ defined in (16) form the transition probabilities, observation probabilities and reward vectors of a two-valued control $\left(\nu_{k} \in\{1,2\}\right)$ and objective

$$
\max _{\nu} \mathbf{E}\left[\sum_{k=0}^{N} \beta^{k} \bar{R}_{\nu_{k}}^{\prime}(p) \pi_{k}\right]
$$

Here, the vector $\pi^{(p)} \in \Pi^{(p)}$ is an information state for this POMDP and evolves according to

$$
\begin{gathered}
\pi_{k+1}^{(p)}=\bar{T}^{(p)}\left(x_{k}^{(p)}, y_{k+1}^{(p)}, \nu_{k}\right), \quad \nu_{k} \in\{1,2\}, \quad \bar{y}_{k+1} \in\left\{1, \ldots, M_{p}+1\right\}, \\
\text { where } \bar{T}^{(p)}\left(x^{(p)}, \bar{y}, \nu\right) \triangleq \frac{\bar{B}_{\nu}^{(p)}(\bar{y})\left(\bar{A}_{\nu}^{(p)}\right)^{\prime} \pi^{(p)}}{\bar{d}^{(p)}\left(x^{(p)}, \bar{y}, \nu\right)}, \quad \bar{d}^{(p)}\left(x^{(p)}, \bar{y}, \nu\right) \triangleq \mathbf{1}_{2\left(\mathcal{N}_{p}+1\right)}^{\prime} \bar{B}_{\nu}^{(p)}(\bar{y})\left(\bar{A}_{\nu}^{(p)}\right)^{\prime} \pi^{(p)},
\end{gathered}
$$

depending on the control $\nu_{k}$ chosen at each time instant. Note that $\nu_{k}=2$ results in $\pi_{k+1}$ attaining the retirement state $z \otimes\left[\begin{array}{c}0_{N_{p}} \\ 1\end{array}\right]$.

The value iteration recursion for optimizing this POMDP over the finite-horizon $N$ is given by

$$
\begin{gathered}
\bar{V}_{k+1}^{(p)}\left(\pi^{(p)}\right)=\max _{\nu \in\{1,2\}}\left[\bar{R}_{\nu}^{\prime}(p) \pi^{(p)}+\beta \sum_{m=1}^{\mu_{p}+1} \bar{V}_{k}^{(p)}\left(\bar{T}^{(p)}\left(x^{(p)}, m, \nu\right)\right) \bar{d}^{(p)}\left(x^{(p)}, m, \nu\right)\right], \quad k=1,2, \ldots, N \\
\bar{V}_{0}^{(p)}(\pi)=\max \left[\bar{R}_{1}^{\prime}(p) \pi^{(p)}, \bar{R}_{2}^{\prime}(p) \pi^{(p)}\right] .
\end{gathered}
$$


Here, $\bar{V}_{k}^{(p)}\left(\pi^{(p)}\right)$ denotes the value function of the dynamic program,

$$
\bar{V}_{k}^{(p)}(\pi) \triangleq \max \mathbf{E}\left[\sum_{t=N-k}^{N} \beta^{t} \bar{R}_{\nu_{t}}^{\prime}(p) \pi_{t} \mid \pi_{N-k}=\pi\right] .
$$

The following result gives a finite-dimensional representation of the Gittins index and is the main result of this section.

THEOREM 3.1. Under the coordinate basis defined in (16), the following three statements hold:

(i) The value function $V_{k}^{(p)}\left(x^{(p)}, M\right)$ in (12) for computing the Gittins index is identically equal to the value function $\bar{V}_{k}^{(p)}\left(\pi^{(p)}\right)$ of the standard POMDP (18).

(ii) At each iteration $k, k=0,1, \ldots, N$, the value function $\bar{V}_{k}^{(p)}\left(\pi^{(p)}\right)$ is piecewise linear and convex and has the finite-dimensional representation

$$
\bar{V}_{k}^{(p)}\left(\pi^{(p)}\right)=\max _{\lambda_{i, k} \in \Lambda_{k}^{(p)}} \lambda_{i, k}^{\prime} \pi^{(p)}
$$

Here, the $2\left(\mathcal{N}_{p}+1\right)$-dimensional vectors $\lambda_{i, k}$ belong to precomputable finite set of vectors $\Lambda_{k}^{(p)}$.

(iii) There always exists a vector in $\Lambda_{k}^{(p)}$, which we shall denote by $\lambda_{1, k}=\left[\begin{array}{lllll}\bar{M} \mathbf{1}_{\mathcal{N}_{p}}^{\prime} & 0 & \mathbf{0}_{\mathcal{N}_{p}}^{\prime} & 0\end{array}\right]^{\prime}$ with optimal control $\nu_{k}=2$.

(iv) Denote the elements of each vector $\lambda_{i, k} \in \Lambda_{k}^{(p)}-\left\{\lambda_{1, k}\right\}$ as

$$
\lambda_{i, k}=\left[\begin{array}{llll}
\lambda_{i, k}^{\prime}(1) & \lambda_{i, k}(2) & \lambda_{i, k}^{\prime}(3) & \lambda_{i, k}(4)
\end{array}\right]^{\prime}, \quad \text { where } \quad \lambda_{i, k}(1), \lambda_{i, k}(3) \in \mathbb{R}^{N_{p}}, \quad \lambda_{i, k}(2), \lambda_{i, k}(4) \in \mathbb{R} .
$$

Then at time $k=N$, for any information state $x^{(p)} \in \mathscr{L}^{(p)}$ of process $p$, the near-optimal Gittins index $\gamma_{N}^{(p)}\left(x^{(p)}\right)$ is given by the finite-dimensional representation (recall $\bar{x}^{(p)}=\left[\begin{array}{c}x^{(p)} \\ 0\end{array}\right]$ )

$$
\begin{gathered}
\gamma_{N}^{(p)}\left(x^{(p)}\right)=\left\{M_{j}: \max _{\lambda_{i, N} \in \Lambda_{N}^{(p)}-\left\{\lambda_{1, N}\right\}} \lambda_{i, N}^{\prime}\left[\begin{array}{c}
\left(M_{j} / \bar{M}\right) \bar{x}^{(p)} \\
\left(1-M_{j} / \bar{M}\right) \bar{x}^{(p)}
\end{array}\right]-M_{j}=0\right\}, \\
\text { where } M_{j}=\frac{\bar{M} \lambda_{j, N}^{\prime}(3) x^{(p)}}{\bar{M}+\left(\lambda_{j, N}(3)-\lambda_{j, N}(1)\right)^{\prime} x^{(p)}}, j=2, \ldots,\left|\Lambda_{N}^{(p)}\right| .
\end{gathered}
$$

REMARK 3.1. Statement 1 of the above theorem shows that the value iteration algorithm (12) for computing the Gittins index $\gamma_{k}^{(p)}\left(x^{(p)}\right)$ is identical to the dynamic programming recursion (18) for optimizing a standard finite-horizon POMDP. Statement 2 says that the finite-horizon POMDP has a finite-dimensional piecewise linear solution, which is characterized by a precomputable finite set of vectors at each time instant. Statement 2 is well known in the POMDP literature and is easily shown by mathematical induction. It was originally proved by Smallwood and Sondik [18], see also Cassandra [2] for a web-based tutorial. There are several linear programming-based algorithms available for computing the finite set of vectors $\Lambda_{k}^{(p)}$ at each iteration $k$, further details are given in $\$ 5$.

Given that we can compute the set of vectors $\Lambda_{N}^{(p)}$, statement 4 gives an explicit formula for the Gittins index $\gamma_{N}^{(p)}\left(x_{k}^{(p)}\right)$, where $x_{k}^{(p)}$ is the information state computed by the $p$ th Bayesian filter at time $k$. We say (22) is an "explicit formula" because it involves maximization over finite sets. That is, given the current information state $x_{k}^{(p)}$ and the set of vectors $\Lambda_{N}^{(p)}$, we only need to compute $M_{j}, j=2, \ldots,\left|\Lambda_{N}^{(p)}\right|$ and then pick one of them according to (22) to obtain the Gittins index $\gamma_{N}^{(p)}\left(x^{(p)}\right)$.

Proof. The proof of the first statement is by mathematical induction. At iteration $k=0$,

$$
\bar{V}_{0}^{(p)}(\pi)=\max \left[\bar{R}_{1}^{\prime}(p) \pi^{(p)}, \bar{R}_{2}^{\prime}(p) \pi^{(p)}\right]=\max \left[(\mathbf{1} \otimes R(p))^{\prime}\left(z \otimes \bar{x}^{(p)}\right), M\right]=V_{0}^{(p)}\left(x^{(p)}, M\right) .
$$

Assume that at time $k, \bar{V}_{k}^{(p)}(\pi)=V_{k}^{(p)}\left(x^{(p)}, M\right)$, and consider (18). Our aim is to show that the right-hand side (RHS) of (18) is the same as the RHS of (12), which would imply that $\bar{V}_{k+1}^{(p)}(\pi)=V_{k+1}^{(p)}\left(x^{(p)}, M\right)$. Note that by construction of the rewards in (16), we have for the terminal state

$$
\bar{V}_{k}^{(p)}\left(z \otimes\left[\begin{array}{c}
\mathbf{0}_{\mathcal{N}_{p}} \\
1
\end{array}\right]\right)=0 \quad k=0,1,2, \ldots
$$


From the definitions of $\pi$ and $\bar{R}_{1}^{\prime}(p) \pi^{(p)}, \bar{R}_{2}^{\prime}(p) \pi^{(p)}$ in (16), it follows that

$$
\bar{R}_{1}^{\prime}(p) \pi^{(p)}=R^{\prime}(p) x^{(p)}, \quad \bar{R}_{2}^{\prime}(p) \pi^{(p)}=M .
$$

Now, consider the terms within the summation of the RHS of (18). Since by our inductive hypothesis, $\bar{V}_{k}^{(p)}(\pi)=$ $V_{k}^{(p)}\left(x^{(p)}, M\right)$, it is easily shown using standard properties of tensor products (recall $\bar{B}_{1}^{(p)}(m), \bar{A}_{1}^{(p)}$ are defined in terms of tensor products in (16)) that for $m=1,2, \ldots, M_{p}$,

$$
\bar{V}_{k}^{(p)}\left(\bar{T}^{(p)}\left(\pi^{(p)}, m, 1\right)\right) \bar{d}^{(p)}\left(\pi^{(p)}, m, 1\right)=V_{k}^{(p)}\left(T^{(p)}\left(x^{(p)}, m\right), M\right) d^{(p)}\left(x^{(p)}, m\right) .
$$

Since $\bar{B}_{1}^{(p)}\left(\Re_{p}+1\right)=\operatorname{diag}\left(\mathbf{0}_{\mathcal{N}_{p}}, 1\right)$ (see (14), (16)) and because of the structure of $\bar{A}_{2}^{(p)}$, it follows that

$$
\begin{gathered}
\bar{V}_{k}^{(p)}\left(\bar{T}^{(p)}\left(\pi^{(p)}, \mu_{p}+1,1\right)\right)=\bar{V}_{k}^{(p)}\left(z \otimes\left[\begin{array}{c}
\mathbf{0}_{\mathcal{N}_{p}} \\
1
\end{array}\right]\right)=0 \quad \forall \pi^{(p)} \in \Pi^{(p)} \\
\bar{V}_{k}^{(p)}\left(\bar{T}^{(p)}\left(\pi^{(p)}, m, 2\right)\right)=\bar{V}_{k}^{(p)}\left(z \otimes\left[\begin{array}{c}
\mathbf{0}_{\mathcal{N}_{p}} \\
1
\end{array}\right]\right)=0 \quad \forall \pi^{(p)} \in \Pi^{(p)}, \quad \forall m \in\left\{1, \ldots, \mu_{p}+1\right\},
\end{gathered}
$$

where $\Pi^{(p)}$ is defined in (17) and the last equality follows from (24). From (25), (26), and (27), it follows that the RHS of (18) is identical to the RHS of (12), implying that $\bar{V}_{k+1}^{(p)}(\pi)=V_{k+1}^{(p)}\left(x^{(p)}, M\right)$.

The third statement follows from (18) and the fact that $\bar{V}_{k}^{(p)}(\pi)$ is piecewise linear and convex. Indeed, from (18), $\bar{V}_{k+1}^{(p)}(\pi)=\max \left[\right.$ piecewise linear segments in $\left.\pi, \bar{R}_{2}^{\prime}(p) \pi\right]$, and hence $\bar{R}_{2}(p)=\left[\begin{array}{lllll}\bar{M} \mathbf{1}_{\mathcal{N}_{p}}^{\prime} & 0 & \mathbf{0}_{\mathcal{N}_{p}}^{\prime} & 0\end{array}\right]^{\prime}$ is one of the elements in $\Lambda_{N+1}^{(p)}$.

The fourth statement can be shown as follows:

$$
\bar{V}_{N}^{(p)}\left(\pi^{(p)}\right)=\max _{\lambda_{i, N} \in \Lambda_{N}^{(p)}} \lambda_{i, N}^{\prime} \pi^{(p)}=\max \left\{\lambda_{1, N}^{\prime} \pi^{(p)}, \max _{\lambda_{i, N} \in \Lambda_{N}^{(p)}-\left\{\lambda_{1, N}\right\}} \lambda_{i, N}^{\prime} \pi^{(p)}\right\} .
$$

Substituting $\lambda_{N, 1}^{\prime} \pi=M$ yields

$$
\bar{V}_{N}^{(p)}\left(\pi^{(p)}\right)=\max \left\{M, \max _{\lambda_{i, N} \in \Lambda_{N}^{(p)}-\left\{\lambda_{1, N}\right\}} \lambda_{i, N}^{\prime} \pi^{(p)}\right\} .
$$

From (13) and the statement 1 of the theorem, the Gittins index is $\gamma_{N}^{(p)}\left(x^{(p)}\right)=\min \left\{M: \bar{V}_{N}^{(p)}\left(\pi^{(p)}\right)=M\right\}$. With the aim of computing $\gamma_{N}^{(p)}\left(x^{(p)}\right)$, let us examine more closely the set $\left\{M: \bar{V}_{N}^{(p)}\left(\pi^{(p)}\right)=M\right\}$. From (28), and using the fact that $\max (a, b)=a \Longrightarrow b \leq a$ for the second equality below yields

$$
\begin{aligned}
\left\{M: \bar{V}_{N}^{(p)}\left(\pi^{(p)}\right)=M\right\} & =\left\{M: \max \left\{M, \max _{\lambda_{i, N} \in \Lambda_{N}^{(p)}-\left\{\lambda_{1, N}\right\}} \lambda_{i, N}^{\prime} \pi^{(p)}\right\}=M\right\} \\
& =\left\{M: \max _{\lambda_{i, N} \in \Lambda_{N}^{(p)}-\left\{\lambda_{1, N}\right\}} \lambda_{i, N}^{\prime} \pi^{(p)} \leq M\right\} .
\end{aligned}
$$

If $\Lambda_{N}^{(p)}-\left\{\lambda_{1, N}\right\}$ is the empty set, then by Lemma 3.1, the Gittins index

$$
\gamma_{N}^{(p)}\left(x^{(p)}\right)=\min \left\{M: \bar{V}_{N}^{(p)}\left(\pi^{(p)}\right)=M\right\}=-B /(1-\beta)
$$

is degenerate. So consider the case where $\Lambda_{N}^{(p)}-\left\{\lambda_{1, N}\right\}$ is nonempty. But $\max _{\lambda_{i, N} \in \Lambda_{N}^{(p)}-\left\{\lambda_{1, N}\right\}} \lambda_{i, N}^{\prime} \pi^{(p)}-M$ is piecewise linear, convex and decreasing in $M$ (see Lemma 3.1), implying that it crosses zero strictly once. Hence there exists a unique

$$
\begin{aligned}
\gamma_{N}^{(p)}\left(x^{(p)}\right)=\min \left\{M: \bar{V}_{N}^{(p)}\left(\pi^{(p)}\right)-M=0\right\} & =\left\{M: \max _{\lambda_{i, N} \in \Lambda_{N}^{(p)}-\left\{\lambda_{1, N}\right\}} \lambda_{i, N}^{\prime} \pi^{(p)}-M=0\right\} \\
& =\left\{M: \max _{\lambda_{i, N} \in \Lambda_{N}^{(p)}-\left\{\lambda_{1, N}\right\}} \lambda_{i, N}^{\prime}\left[\begin{array}{c}
(M / \bar{M}) \bar{x}^{(p)} \\
(1-M / \bar{M}) \bar{x}^{(p)}
\end{array}\right]-M=0\right\},
\end{aligned}
$$


where in the last equality above we have used (16) to substitute $\pi^{(p)}=[M / \bar{M}, 1-M / \bar{M}]^{\prime} \otimes \bar{x}^{(p)}$. Let $M_{j}$, $j=2, \ldots,\left|\Lambda_{N}^{(p)}\right|$ denote the solution of the $\left|\Lambda_{N}^{(p)}\right|-1$ algebraic equations

$$
\lambda_{j, N}^{\prime}\left[\begin{array}{c}
(M / \bar{M}) \bar{x}^{(p)} \\
(1-M / \bar{M}) \bar{x}^{(p)}
\end{array}\right]-M=0 .
$$

Using the structure of $\lambda_{j, N}$ in (21) to solve the above equation for $M_{j}$ yields

$$
M_{j}=\frac{\bar{M} \lambda_{j, N}^{\prime}(3) x^{(p)}}{\bar{M}+\left(\lambda_{j, N}(3)-\lambda_{j, N}(1)\right)^{\prime} x^{(p)}}, \quad j=2, \ldots,\left|\Lambda_{N}^{(p)}\right| .
$$

Given these values for $M_{j}, j=2, \ldots,\left|\Lambda_{N}^{(p)}\right|$, the Gittins index is the choice of $M_{j}$ that satisfies (29). That is,

$$
\gamma_{N}^{(p)}\left(x^{(p)}\right)=\left\{M_{j}: \max _{\lambda_{i, N} \in \Lambda_{N}^{(p)}-\left\{\lambda_{1, N}\right\}} \lambda_{i, N}^{\prime}\left[\begin{array}{c}
\left(M_{j} / \bar{M}\right) \bar{x}^{(p)} \\
\left(1-M_{j} / \bar{M}\right) \bar{x}^{(p)}
\end{array}\right]-M_{j}=0\right\} .
$$

3.2. Numerical algorithms. In the previous section, we showed that the Gittins index for each process $p$, can be computed by solving a POMDP associated with process $p$. Thus, instead of solving a POMDP comprising of $\mathcal{N}_{1} \times \cdots \times \mathcal{N}_{P}$ states and $P$ actions (which would be the brute force solution), because of the bandit structure, we only need to solve $P$ independent POMDPs, each comprising of $2\left(\mathcal{N}_{p}+1\right)$ states and two actions. This makes the POMDP multiarmed bandit problem tractable. However, it should be noted that even with the bandit formulation, solving a $2\left(\mathcal{N}_{p}+1\right)$ state, $\mu_{p}+1$ observation POMDP can still be expensive even for small $\mathcal{N}_{p}$ if $M_{p}$ is large. As mentioned in $\S 1$, POMDPs are PSPACE hard problems-in worst case, the number of vectors in $\Lambda_{k}$ can grow exponentially with $k$.

Given the finite-dimensional representation of the Gittins index in (22) of Theorem 3.1, there are several linear programming-based algorithms in the POMDP literature such as Sondik's algorithm, Monahan's algorithm, Cheng's algorithm, and the Witness algorithm (Cassandra [2]) that can be used to compute the finite set of vectors $\Lambda_{N}^{(p)}$ depicted in Equation (20). In the numerical examples below, we used the "incremental prune" algorithm recently developed in the artificial intelligence community by Cassandra et al. in 1997 (Cassandra et al. [4], Cassandra [3])— the C++ code can be freely downloaded from the website (Cassandra [2]).

3.3. Suboptimal algorithm based on Lovejoy's [13] approximation. Lovejoy [13] proposed an ingenious sub-optimal algorithm for POMDPs-here we adapt it to computing the Gittins index of the POMDP bandit. It is obvious that by considering only a subset of the piecewise linear segments that characterize $\bar{V}_{k}^{(p)}(\pi)$ and discarding the other segments, one can reduce the computational complexity. This is the basis of Lovejoy's [13] lower-bound approximation. Lovejoy's [13] algorithm operates as follows: Initialize $\bar{\Lambda}_{0}^{\prime}=\Lambda_{0}^{(p)}$, i.e., according to (23).

Step 1. Given a set of vectors $\bar{\Lambda}_{k}^{(p)}$, construct the set $\tilde{\Lambda}_{k}^{(p)}$ by pruning $\bar{\Lambda}_{k}^{(p)}$ as follows: Pick any $R$ points, $\pi_{1}, \pi_{2}, \ldots, \pi_{R}$ in the information state simplex $\Pi^{(p)}$. (In the numerical examples below, we picked the $R$ points based on a uniform Freudenthal triangularization of $\Pi^{(p)}$, see Lovejoy [13] for details.) Then set $\tilde{\Lambda}_{k}^{(p)}=$ $\left\{\arg \max _{\lambda \in \bar{\Lambda}_{k}^{(p)}} \lambda^{\prime} \pi_{r}, r=1,2, \ldots, R\right\}$.

Step 2. Given $\tilde{\Lambda}_{k}^{(p)}$, compute the set of vectors $\bar{\Lambda}_{k+1}^{(p)}$ using a standard POMDP algorithm.

Step 3. $k \rightarrow k+1$.

Notice that $\widetilde{V}_{k}^{(p)}(\pi)=\max _{\lambda \in \tilde{\Lambda}_{k}^{(p)}} \lambda^{\prime} \pi$ is represented completely by $R$ piecewise linear segments. Lovejoy [13] shows that for all $k, \bar{V}_{k}^{(p)}(\pi)$ is a lower bound to the optimal value function $V_{k}^{(p)}(\pi)$, i.e., $V_{k}^{(p)}(\pi) \geq$ $\bar{V}_{k}^{(p)}(\pi)$ for all $\pi \in \mathscr{P}$. Lovejoy's algorithm gives a suboptimal scheduling policy at a computational cost of no more than $R$ evaluations per iteration $k$. Lovejoy [13] also provides a constructive procedure for computing an upper bound to $\lim \sup _{\pi \in \mathscr{P}}\left|V_{k}^{(p)}(\pi)-\bar{V}_{k}^{(p)}(\pi)\right|$. In $\S 5$, it is shown that Lovejoy's approximation yields excellent performance.

4. Structural results for monotone Gittins index. The value iteration-based solutions for computing the Gittins index given in the previous section work for small $\mathcal{N}$ and $M$. However, for large $\mathcal{N}$ and $\mathcal{M}$, solving each individual POMDP to compute the Gittins index can become computationally intractable. In this section, we show that under reasonable conditions on the rewards, transition matrix and observation probabilities, the Gittins index is monotone increasing in the information state (with respect to the MLR order). This means that if the 
information states of the $P$ processes at a given time instant are MLR comparable, the optimal policy is to pick the process with the largest information state. This is trivial to implement and makes the solution practically useful.

However, because the MLR is a partial ordering, not all information state trajectories are MLR comparable. Therefore we propose a linear approximation to the Gittins index and give a necessary and sufficient condition for it to be MLR increasing. The resulting best linear approximation to the Gittins index yields a computationally simple policy that coincides with the optimal policy when the states are MLR comparable. We then give a stochastic approximation algorithm to estimate the best linear approximation of the Gittins index. Finally, we present another approximation where non-MLR comparable information states are projected onto the nearest MLR comparable state. This turns out to be a convex optimization problem and can be solved efficiently.

4.1. Characterization of monotone Gittins index. In this section, we assume that all the $P$ processes evolve with identical dynamics, i.e., $A^{(p)}, B^{(p)}, R(p), \mathscr{X}^{(p)}, \Omega_{p}$ are identical. So we omit the index $p$ for them. Our goal is to give conditions on the parameters $A, B, R$, so that the Gittins index $\gamma(x)$ is monotone in information state $x$ with respect to the MLR ordering.

For future reference, we collect the following standard definitions.

Definition 4.1 (MLR Ordering and First-Order Stochastic Dominance (Muller and Stoyan (13)). Let $x^{(1)} \in \mathscr{X}, x^{(2)} \in \mathscr{X}$ be any two information state vectors.

(i) Then $x^{(1)}$ is greater than $x^{(2)}$ with respect to the MLR ordering-denoted as $x^{(1)} \geq_{r} x^{(2)}$ if

$$
x^{(1)}(i) x^{(2)}(j) \leq x^{(2)}(i) x^{(1)}(j), \quad i<j, \quad i, j \in\{1, \ldots, \mathcal{N}\}
$$

(ii) A function $f(\cdot)$ is MLR increasing if for all $x^{(1)}, x^{(2)} \in \mathscr{X}, x_{1} \geq_{r} x_{2}$ implies $f\left(x^{(1)}\right) \geq f\left(x^{(2)}\right)$.

(iii) Let $x^{(1)}, x^{(2)}, \ldots, x^{(P)}$ denote $P$ information states. Then they are said to be MLR comparable if for any $p, \tilde{p} \in\{1, \ldots, P\}$, either $x^{(p)} \leq_{r} x^{(\tilde{p})}$ or $x^{(p)} \geq_{r} x^{(\tilde{p})}$.

(iv) Given $P$ MLR comparable information states, denote the largest information state (wrt MLR ordering) as $\max \left\{x^{(1)}, \ldots, x^{(P)}\right\}$ with index $\arg \max \left\{x^{(1)}, \ldots, x^{(P)}\right\}$.

(v) $x^{(1)}$ is greater than $x^{(2)}$ with respect to first-order stochastic dominance-denoted as $x^{(1)}>_{s} x^{(2)}$ if $\sum_{i=j}^{\mathcal{N}} x^{(1)}(i) \geq \sum_{i=j}^{\mathcal{N}} x^{(2)}(i)$ for all $j \in\{1, \ldots, \mathcal{N}\}$.

(vi) Equivalently, $x^{(1)}>_{s} x^{(2)}$ iff $\sum_{i=1}^{\mathcal{N}} f(i) x^{(1)}(i) \geq \sum_{i=1}^{\mathcal{N}} f(i) x^{(2)}(i)$ for all increasing functions $f$.

The main result of the section is the following (recall we omit index $p$ for $T, d, R$ ).

THEOREM 4.1. Consider the following assumptions for each process $p \in\{1, \ldots, P\}$ in the POMDP multiarmed bandit.

Assumption (A1). Rewards satisfy $R(i) \leq R(i+1)$.

Assumption (A2). Transition probability matrix A is TP2, i.e., all its second-order minors are nonnegative. That is, determinants

$$
\left|\begin{array}{ll}
a_{i_{1} j_{1}} & a_{i_{1} j_{2}} \\
a_{i_{2} j_{1}} & a_{i_{2} j_{2}}
\end{array}\right| \geq 0 \quad \text { for } i_{2} \geq i_{1}, \quad j_{2} \geq j_{1} .
$$

Assumption (A3). Symbol probabilities satisfy $\left(b_{i, m}\right)_{m \in M} \geq_{r}\left(b_{i+1, m}\right)_{m \in \mathscr{M}}$ for $i=1, \ldots, \mathcal{N}-1$.

Then, the Gittins index $\gamma(x)$ is MLR increasing (see Definition 4.1(ii)). Therefore, if the information states $x_{k}^{(p)}$ of the P projects are MLR comparable, then the optimal policy $\mu^{*}$ defined in (9) is opportunistic:

$$
u_{k}=\mu^{*}\left(x_{k}^{(1)}, \ldots, x_{k}^{(P)}\right)=\underset{p \in\{1, \ldots, P\}}{\arg \max } x_{k}^{(p)} .
$$

Proof. We begin with the following lemma (see Lovejoy [12] for proof).

LEMMA 4.1 (LoveJoy [12]). With the Bayesian filter $T(x, m)$ and normalization term $d(x, m)$ defined in (5),

(i) (A1) implies $R^{\prime} x$ is MLR increasing in $x$

(ii) (A2) and (A3) imply $T(x, m)$ is MLR increasing in $m$

(iii) (A2) and (A3) imply $T(x, m)$ is MLR increasing in $x$

(iv) (A3) and (A3) imply $(d(x, m))_{m \in M}$ is first-order stochastically increasing in $x$.

We prove first that $V(x, M)$ is MLR increasing in $x$ via mathematical induction on the value iteration algorithm (12). $V_{0}(x, M)$ can be chosen arbitrarily to be MLR increasing in $x$. Now, assume $x_{1} \geq_{r} x_{2}$ and $V_{k}\left(x_{1}, M\right)>V_{k}\left(x_{2}, M\right)$, i.e, $V_{k}(x, M)$ is MLR increasing in $x$. Then Lemma 4.1(i) implies $R^{\prime} x_{1}>R^{\prime} x_{2}$. Next, 
since $T(x, m)$ is MLR increasing in $m$ (Lemma 4.1(ii)), and $V_{k}(\cdot)$ is MLR increasing by hypothesis, the vector $\left[V_{k}(T(x, 1), M), V_{k}(T(x, 2), M), \ldots, V_{k}(T(x, M), M)\right]$ has nondecreasing components. From Lemma 4.1(iv), $\left(d\left(x_{1}, m\right)\right)_{m \in M}>_{s}\left(d\left(x_{2}, m\right)\right)_{m \in \Perp}$. Therefore, by Definition 4.1(vi),

$$
\sum_{m=1}^{M} V_{k}\left(T\left(x_{1}, m\right), M\right) d\left(x_{1}, m\right) \geq \sum_{m=1}^{M} V_{k}\left(T\left(x_{1}, m\right), M\right) d\left(x_{2}, m\right) \geq \sum_{m=1}^{M} V_{k}\left(T\left(x_{2}, m\right), M\right) d\left(x_{2}, m\right),
$$

where the last inequality follows Lemma 4.1(iii). Therefore

$$
R^{\prime} x_{1}+\sum_{m=1}^{M} V_{k}\left(T\left(x_{1}, m\right), M\right) d\left(x_{1}, m\right) \geq R^{\prime} x_{2}+\sum_{m=1}^{M} V_{k}\left(T\left(x_{2}, m\right), M\right) d\left(x_{2}, m\right) .
$$

Substituting this into the value iteration algorithm (12) proves that $V_{k+1}(x, M)$ is MLR increasing in $x$. Finally, since $V_{k}(x, M) \rightarrow V(x, M)$ uniformly as $k \rightarrow \infty, V(x, M)$ is MLR increasing in $x$.

Given that the value function $V(x, M)$ is MLR increasing in $x$, we can now characterize the Gittins index. Recall $\gamma(x)=\min \{M: V(x, M)-M=0\}$. Suppose $x^{(1)} \geq_{r} x^{(2)}$. This implies $V\left(x^{(1)}, M\right) \geq V\left(x^{(2)}, M\right)$ for all $M$. So $V\left(x^{(1)}, \gamma\left(x^{(2)}\right)\right)-\gamma\left(x^{(2)}\right) \geq V\left(x^{(2)}, \gamma\left(x^{(2)}\right)\right)-\gamma\left(x^{(2)}\right)=0$. Since $V(x, M)-M$ is decreasing in $M$, it follows from the previous inequality that the point $\min \left\{M: V\left(x^{(1)}, M\right)-M=0\right\}>\min \left\{M: V\left(x^{(2)}, M\right)-M=0\right\}$. So $\gamma\left(x^{(1)}\right) \geq \gamma\left(x^{(2)}\right)$.

4.1.1. Discussion. When the $P$ information states are MLR comparable, then Theorem 4.1 says that the optimal policy is to pick the process with the largest MLR state, i.e., the optimal policy is opportunistic (other terms used are "greedy" or "myopic"). Of course, because MLR is a partial ordering, in general, the $P$ information states need not be MLR comparable. If they are MLR comparable, then Theorem 4.1 applies.

Several examples for $\mathcal{N} \geq 2$ can be constructed that yield trajectories of information states that are MLR comparable. Here are three simple examples.

EXAMPLE 1. If each process has state-space size $\mathcal{N}=2$, then all information states are MLR comparable and so the above theorem can be used to determine the optimal policy for arbitrarily large observation symbol size $\mathcal{M}$. (The MLR ordering coincides with first-order stochastic dominance for $\mathcal{N}=2$.)

EXAmple 2. Suppose $\left(b_{i, m}\right)$ is a bidiagonal matrix. Then if $x_{0}^{(p)}$ is a unit indicator vector, then all subsequent information states are MLR comparable (because all information states comprise of two consecutive nonzero elements and the rest are zero elements).

Example 3. Suppose $b_{i . m}=1 / M$ for all $i, m$. Suppose all processes have the same initial information state $x_{0}$ and pick $A$ such that either $A^{\prime} x_{0} \geq_{r} x_{0}$ or $A^{\prime} x_{0} \leq_{r} x_{0}$. Then from Kijima [6, Theorem 3.13] if $A$ is TP2, all information states are MLR comparable. So if $x_{0}$ is a unit indicator vector and $A$ is TP2, then all information states are MLR comparable.

In the above three examples, under (A1), (A2), (A3), the optimal policy is opportunistic.

4.2. Suboptimal approximations to the Gittins index. In the previous section, we showed that under Assumptions (A1), (A2), (A3), when the $P$ information states $x^{(1)}, \ldots, x^{(P)}$ are MLR comparable, then the optimal policy is opportunistic: pick the largest information state (with respect to the MLR ordering). However, since for $\mathcal{N} \geq 3$, MLR is a partial ordering, the $P$ information states $x^{(1)}, \ldots, x^{(P)}$ are not necessarily MLR comparable at each time instant. When the trajectories of information states are not MLR comparable, the following approximations can be used. Naturally, throughout this subsection, we are only interested in $\mathcal{N} \geq 3$ since $\mathcal{N}=2$ is completely orderable.

4.2.1. Projection to nearest MLR state. Assume at time instant $k$, the information states of all $P$ processes are MLR comparable. Let $\sigma(1), \ldots, \sigma(P)$ denote the permutation of $(1, \ldots, P)$, so that $x_{k}^{\sigma(1)} \geq_{r} x_{k}^{\sigma(2)} \geq_{r} \cdots \geq_{r} x_{k}^{\sigma(P)}$. From the above theorem, the optimal action is $u_{k}=\sigma(1)$. But the updated information state $x_{k+1}^{\sigma(1)}$ may not be MLR comparable with the other $P-1$ information states. So we project $x_{k+1}^{\sigma(1)}$ to the nearest information state denoted $\bar{x}$ in the simplex $\mathscr{X}$ that is MLR comparable with the other $P-1$ information states. That is, at time $k+1$, we solve the following $P$ optimization problems:

$$
\begin{array}{rlll}
\text { Compute } J\left(\bar{x}^{(1)}\right) & =\min _{\bar{x} \in \mathscr{C}}\left\|\bar{x}-x_{k+1}^{\sigma(1)}\right\| & \text { subject to } & \bar{x} \geq_{r} x_{k}^{\sigma(2)} \\
J\left(\bar{x}^{(p)}\right) & =\min _{\bar{x} \in \mathscr{C}}\left\|\bar{x}-x_{k+1}^{\sigma(1)}\right\| & \text { subject to } & x_{k}^{\sigma(p)} \geq_{r} \bar{x} \geq_{r} x_{k}^{\sigma(p+1)}, \quad p=2, \ldots, P-1 \\
J\left(\bar{x}^{(P)}\right) & =\min _{\bar{x} \in \mathscr{L}}\left\|\bar{x}-x_{k+1}^{\sigma(1)}\right\| & \text { subject to } & x_{k}^{\sigma(P)} \geq_{r} \bar{x} .
\end{array}
$$


Here, $\|\cdot\|$ denotes some norm, and $J, \bar{x}_{p}$ denote, respectively, the minimizing value and minimizing solution of each of the problems. Finally, set $x_{k+1}^{\sigma(1)}=\arg \min _{\bar{x}_{p}} J\left(\bar{x}_{p}\right)$.

The above $P$ problems are straightforwardly shown to be a convex optimization problems and can be solved efficiently in real time. Thus, all the information states at time $k+1$ are now MLR comparable, the optimal action $u_{k+1}$ is chosen as the index of the largest information state, and so on.

4.3. Best linear approximation for Gittins index. Recall from (22) that the Gittins index $\gamma(x)$ is a rational function of the information state $x$. Suppose we construct a linear approximation to the Gittins index $\gamma(x)$ as $\gamma_{\theta}(x)=\theta^{\prime} x$. Here, $\theta \in \mathbb{R}^{\mathcal{N}}$ denotes the coefficient vector of the linear approximated Gittins index $\gamma_{\theta}(x)$. Then the scheduling policy becomes

$$
\mu_{\theta}\left(x^{(1)}, x^{(2)}, \ldots, x^{(P)}\right)=\underset{p \in\{1, \ldots, P\}}{\arg \max } \theta^{\prime} x^{(p)} .
$$

Assuming (A3), (A3), and (A3) hold, we know from Theorem 4.1 that the true Gittins index $\gamma(x)$ is MLR increasing in $x$. What constraints on the coefficient vector $\theta$ ensures that the linear approximation $\gamma_{\theta}(x)$ is also MLR increasing in $x$ ?

TheOREM 4.2. For general $\mathcal{N}$, a sufficient condition on $\theta$ for $\gamma_{\theta}(x)$ to be MLR increasing (see Definition 4.1(ii)) is that the elements of $\theta$ are nondecreasing. For the special case $\mathcal{N}=3$, the elements of $\theta$ being nondecreasing is necessary and sufficient for $\gamma_{\theta}(x)$ to be MLR increasing.

Proof. The first claim follows straightforwardly since $x^{(1)} \geq_{r} x^{(2)} \Rightarrow x^{(1)}>_{s} x^{(2)} \Rightarrow \theta^{\prime} x^{(1)}>\theta^{\prime} x^{(2)}$ for any increasing $\theta$ (Definition 4.1(vi)).

To prove the "only if" for $\mathcal{N}=3$ : Let $e_{1}=(1,0,0), e_{3}=(0,0,1)$. It is easily checked that if $\mathcal{N}=3$, then $x^{(1)} \geq_{r} x$ iff $x^{(1)}=\alpha\left[\epsilon_{1} e_{1}+\left(1-\epsilon_{1}\right) x\right]+(1-\alpha)\left[\epsilon_{3} e_{3}+\left(1-\epsilon_{3}\right) x\right]$, where $\alpha \in[0,1], \epsilon_{3} \in[0,1]$, and $0 \geq \epsilon_{1} \geq$ $1-\min \{1 /(1-\alpha x(1)), 1 / \alpha x(2), 1 / \alpha x(3)\}$. (These constraints ensure that $x^{(1)}$ is a valid information state.) So $\theta^{\prime} x^{(1)}>\theta^{\prime} x^{(2)}$ implies

$$
\theta^{\prime} x^{(2)}\left(\alpha\left(\epsilon_{1}-\epsilon_{3}\right)+\epsilon_{3}\right) \leq \alpha \epsilon_{1} \theta(1)+(1-\alpha) \epsilon_{3} \theta(3), \quad \forall x^{(2)} \in \mathscr{X}, \quad \forall \alpha \in[0,1] .
$$

Choosing $x^{(2)}=e_{1}$ yields $\theta(1) \leq \theta(3)$. Choosing $x^{(2)}=e_{2}, \alpha=1$, yields $\theta(2) \geq \theta(1)$. Choosing $x^{(2)}=e_{2}, \alpha=0$ yields $\theta(2) \leq \theta(3)$.

Consider now optimizing the coefficient $\theta$ to maximize the reward by solving the following stochastic optimization problem:

$$
\begin{aligned}
& \text { Compute } \mu_{\theta^{*}}(\pi), \quad \text { where } \theta^{*}=\max _{\theta \in \mathbb{R}^{N}} \mathbf{E}\left[\sum_{k=0}^{\infty} \beta^{k} R^{\prime}\left(u_{k}\right) x_{k}^{\left(u_{k}\right)}\right], \text { where } u_{k}=\mu_{\theta}\left(x^{(1)}, \ldots, x^{(p)}\right) \\
& \text { subject to constraints } \quad \theta(1)=0, \quad \theta(\mathcal{N})=1, \quad \theta(i) \leq \theta(i+1), \quad i=2,3, \ldots, \mathcal{N}-1 .
\end{aligned}
$$

(Since $\theta(1)$ is the smallest element, and $\theta(\mathcal{N})$ is the largest element, we set them to zero and one without loss of generality as the policy $\arg \max _{p} \theta^{\prime} x^{(p)}$ is invariant to scaling and translation by constants.)

Remark 4.1. For any $\mathcal{N} \geq 3$, if the $P$ information states $x^{(1)}, \ldots, x^{(P)}$ are MLR comparable, then the linear approximated Gittins index $\gamma_{\theta^{*}}(x)$ yields the optimal policy $\mu^{*}\left(x^{(1)}, \ldots, x^{(P)}\right)$ by Theorem 4.2. For the case $\mathcal{N}=3$, since $\theta(1) \leq \theta(2) \leq \theta(3)$ is both necessary and sufficient for $\gamma(x)$ to be monotone (Theorem 4.2), we can say more. In this case, $\theta^{*}$ qualifies as the best linear MLR approximation to the Gittins index. That is, $\gamma_{\theta^{*}}(x)$ is the best linear approximation amongst the class of MLR increasing functions.

Since it is impossible to analytically compute the optimal parameter $\theta^{*}$, we resort to simulation-based optimization to estimate $\theta^{*}$ in (37). The simulation-based stochastic optimization problem is formulated as

$$
\text { compute } \mu_{\theta^{*}}(\pi), \quad \text { where } \theta^{*}=\max _{\theta \in \mathbb{R}^{N}} \mathbf{E}\left\{J_{n}(\theta)\right\}, \quad \text { subject to constraint (37) }
$$

Here, $n=1,2, \ldots$ denotes the index of an independent simulation batches and in each batch $n, J_{n}(\theta)$ is an asymptotically unbiased estimate of the above reward obtained via simulation as

$$
J_{n}(\theta)=\sum_{k=0}^{N} \beta^{k} R^{\prime}\left(u_{k}\right) x_{k}^{\left(u_{k}\right)}, \quad u_{k}=\mu_{\theta}\left(x^{(1)}, \ldots, x^{(p)}\right) .
$$


The horizon $N$ is chosen as a large positive integer. We can nicely convert the constrained stochastic optimization problem (38) into an equivalent unconstrained using spherical coordinates: For any $\phi \in \mathbb{R}^{\mathcal{N}-2}$ with elements denoted $\phi(1), \ldots, \phi(\mathcal{N}-2)$, define the coefficient vector $\theta^{\phi}$ parameterized by $\phi$ as

$$
\begin{gathered}
\theta^{\phi}(1)=0 ; \quad \theta^{\phi}(i)=\sum_{j=1}^{i-1} \Delta^{2}(j), \quad \text { for } i=2, \ldots, \mathcal{N}-1, \quad \text { where } \\
\Delta(i)=\cos \phi(i) \prod_{l=1}^{i-1} \sin \phi(l), \quad i=1,2, \ldots, \mathcal{N}-3 \\
\Delta(\mathcal{N}-2)=\sin (\phi(\mathcal{N}-3)) \prod_{l=1}^{\mathcal{N}-4} \sin \phi(l) .
\end{gathered}
$$

Since $\Delta^{2}(j)$ are nonnegative clearly, $\theta^{\phi}(i) \geq \theta^{\phi}(i-1)$. Also, it is straightforward to show that spherical coordinates satisfy $\sum_{i=1}^{\mathcal{N}-2} \Delta^{2}(i)=1$. For example, for $\mathcal{N}=3, \phi=\phi(1)$ reduces to the familiar case, $\theta^{\phi}(1)=0$, $\theta^{\phi}(2)=\cos ^{2} \phi(1), \theta^{\phi}(3)=\cos ^{2} \phi(1)+\sin ^{2} \phi(1)=1$.

Thus the constrained stochastic optimization problem (38) is equivalent to the following unconstrained stochastic optimization problem:

$$
\text { compute } \mu_{\theta^{\phi^{*}}}\left(x^{(1)}, \ldots, x^{(P)}\right), \quad \text { where } \phi^{*}=\max _{\phi \in \mathbb{R}^{N-2}} \mathbf{E}\left\{J_{n}\left(\theta^{\phi}\right)\right\}
$$

4.4. Simulation-based stochastic approximation algorithm for estimating linear approximation to monotone Gittins index. Below, we present a policy gradient algorithm, which generates a sequence of estimates $\hat{\phi}_{n}$ (and thus $\hat{\theta}_{n} \triangleq \theta^{\hat{\phi}_{n}}$ ), $n=1,2, \ldots$, that converges to a local maximum of the best MLR increasing linear Gittins index $\theta^{\phi^{*}} x$, with policy $\mu_{\theta^{\phi^{*}}}(\cdot)$ given by (36).

Assume the parameters satisfy Assumptions (A1), (A2), (A3). Hence the optimal policy $\mu^{*}(x)$ is opportunistic (by Theorem 4.1). Below, $\epsilon_{n}$ denotes a decreasing step size for the stochastic approximation algorithm.

Step 1 . Choose initial coefficients $\hat{\theta}_{0}$ and use policy $\mu_{\hat{\theta}_{0}}$.

Step 2. For iterations, $n=0,1,2, \ldots$.

(i) Evaluate sample reward $J_{n}\left(\theta^{\hat{\phi}_{n}}\right)$ via (39) and gradient estimate $\widehat{\nabla}_{\phi} J_{n}\left(\theta^{\hat{\phi}_{n}}\right)$ (see discussion below) using policy $\mu_{\theta_{\hat{\phi}_{n}}}$ defined in (36).

(ii) Update threshold coefficients $\theta^{\hat{\phi}_{n}}$ according to (40), where $\hat{\phi}_{n}$ is updated via stochastic approximation algorithm

$$
\hat{\phi}_{n+1}=\hat{\phi}_{n}+\epsilon_{n+1} \hat{\nabla}_{\phi} J_{n}\left(\theta^{\hat{\phi}_{n}}\right), \quad \epsilon_{n}=\epsilon /(n+1+s)^{\kappa}, \quad 0.5<\kappa<1, \text { and } \epsilon, s>0 .
$$

Because stochastic gradient algorithms converge to local optima, it is necessary to try several initial conditions, and pick the best threshold. Since the explicit dependence of $J_{n}(\theta)$ on $\theta$ is not known, it is not possible to compute $\nabla_{\phi} J_{n}\left(\theta^{\phi}\right)$ in (42) analytically. We need to use gradient estimation to compute an estimate $\widehat{\nabla}_{\phi} J_{n}\left(\theta^{\hat{\phi}_{n}}\right)$. Gradient estimation can be performed using the finite-difference estimator described below or a more sophisticated gradient estimation algorithm such as measured valued differentiation (Krishnamurthy et al. [8]). Below we use the simultaneous perturbation stochastic approximation (SPSA) algorithm (Spall [19]) because of its computational efficiency and excellent numerical properties.

The SPSA algorithm (Spall [19]) picks a single random direction $\omega_{n}$ along which direction the derivative is evaluated at each batch $n$. Unlike the well-known Kiefer-Wolfowitz finite-difference algorithm (Spall [19]), the main advantage of SPSA is that to evaluate the gradient estimate $\widehat{\nabla}_{\phi} J_{n}$ requires only two POMDP simulations, i.e., the number of evaluations is independent of the dimension of the parameter vector $\phi$. We refer the reader to Spall [19] and www.jhuapl.edu/SPSA/ for details of the SPSA algorithm. The SPSA algorithm uses the following gradient estimate $\widehat{\nabla}_{\phi} J_{n}$ in the stochastic gradient algorithm (42):

$$
\widehat{\nabla}_{\phi} J_{n}=\frac{J_{n}\left(\theta^{\hat{\phi}_{n}+\mu_{n} \omega_{n}}\right)-J_{n}\left(\theta^{\hat{\phi}_{n}-\mu_{n} \omega_{n}}\right)}{2 \mu_{n}} \omega_{n}, \quad \text { where } \omega_{n}(i)=\left\{\begin{array}{ll}
-1 & \text { with probability } 0.5 \\
+1 & \text { with probability 0.5. }
\end{array}, \mu_{n}=\frac{\mu}{(n+1)^{\gamma}} .\right.
$$

Here, the $\mathcal{N}-2$-dimensional vector $\omega_{n}$ with random elements $\omega_{n}(i), i=1, \ldots, \mathcal{N}-2$ denotes the random direction chosen by the SPSA algorithm. Typically, $\gamma$ in (43) and $\kappa$ in (42) are chosen so that $\gamma<\kappa$ (see Spall [19]). It can be shown using the techniques in Kushner and Yin [10] that under suitable regularity conditions, the sequence $\left\{\hat{\theta}_{n}\right\}$ generated by the stochastic approximation algorithm converges as $n \rightarrow \infty$ to a local optimum of $\mathbf{E}\left\{J_{n}(\theta)\right\}$ (defined in (38)) with probability one. 


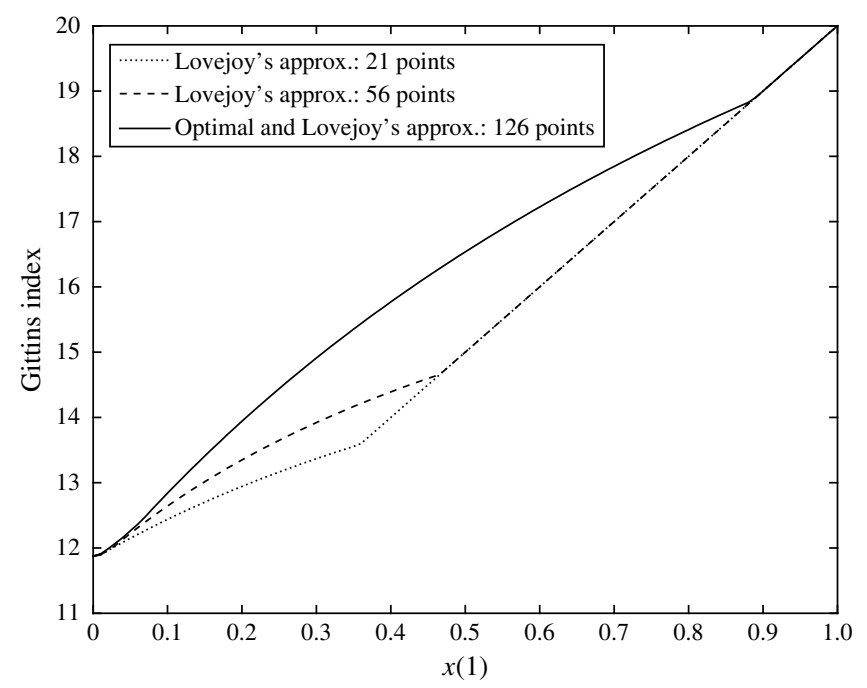

FIGURE 1. Gittins indices for $\mathcal{N}=2, M=2$ computed using Lovejoys's algorithm and optimal Gittins index for horizon $N=20$.

5. Numerical examples. Here, we present numerical examples for computing the Gittins index of POMDP multiarmed bandits using the algorithms presented in this paper. When the state $s_{k}^{(p)}$ of each process evolves according to a two or three state Markov chain, the Gittins index of each process can be graphically illustrated, meaning that a complete discussion of the algorithm behavior can be given.

5.1. Value iteration algorithm for computing Gittins index. We used the POMDP program from the website (Cassandra [2]) to compute the set of vectors $\Lambda_{N}$, for horizon $N=20$. The POMDP program allows the user to choose from several available algorithms. We used the "incremental pruning" algorithm developed by Cassandra et al. [4] in 1997. This is currently one of the fastest known algorithms for solving POMDPs (see Cassandra [2] for details).

We chose parameters

$$
\mathcal{N}=2, \quad M=2, \quad \beta=0.9, \quad A=\left[\begin{array}{ll}
0.7 & 0.3 \\
0.1 & 0.9
\end{array}\right], \quad B=\left[\begin{array}{ll}
0.1 & 0.9 \\
0.8 & 0.2
\end{array}\right], \quad R=\left[\begin{array}{l}
2 \\
1
\end{array}\right] .
$$

A numerical resolution of $\epsilon=5 \times 10^{-3}$ yields 106 vectors for $\Lambda_{20}$. Using these computed vectors, the Gittins index $\gamma_{N}(x)$ computed using (22) are plotted in Figure 1. (Because $\mathcal{N}=2$, and $x(1)+x(2)=1$, it suffices to plot $\gamma_{N}(x)$ versus $x(1)$.)

5.2. Lovejoy's suboptimal algorithm for computing Gittins index. Although the above computation of the Gittins indices is offline, it takes substantial computational time. This motivates the use of Lovejoy's suboptimal algorithm of $\$ 3.2$ to compute the Gittins indices. Figure 1 also illustrates Lovejoy's approximation for grid sizes $R=21$ points (which yielded $\tilde{\lambda}_{20}$ comprising six vectors), $R=56$ points (which yielded $\tilde{\lambda}_{20}$ comprising 13 vectors) and $R=126$ points (which yielded $\tilde{\lambda}_{20}$ comprising 20 vectors), respectively. As can be seen, for grid sizes of 126 and above, the computed Gittins index coincides with the optimal value iteration algorithm. For smaller grid sizes (21 and 56 points), the approximation is as reasonable as can be seen from Figure 1.

For another example, we chose

$$
\mathcal{N}=3, \quad M=3, \quad \beta=0.99, \quad A=\left[\begin{array}{ccc}
0.9 & 0.1 & 0 \\
0.1 & 0.8 & 0.1 \\
0 & 0.1 & 0.9
\end{array}\right], \quad B=A, \quad R=\left[\begin{array}{l}
1 \\
2 \\
3
\end{array}\right] .
$$

For an $R=36$ point uniform triangularization of the information state space, Figure 2 shows the computed Gittins indices using Lovejoy's algorithm. $\tilde{\Lambda}_{20}$ has 12 vectors.

5.3. MLR monotone Gittins index and linear approximations. Since the above POMDP bandit model satisfies Assumptions (A1), (A2), (A3), the Gittins index $\gamma(x)$ is monotone increasing with respect to the MLR order. (For example, in Figure 2, $\gamma\left(e_{1}\right)=177.607<\gamma\left(e_{2}\right)=230.279<\gamma\left(e_{3}\right)=300$.) 


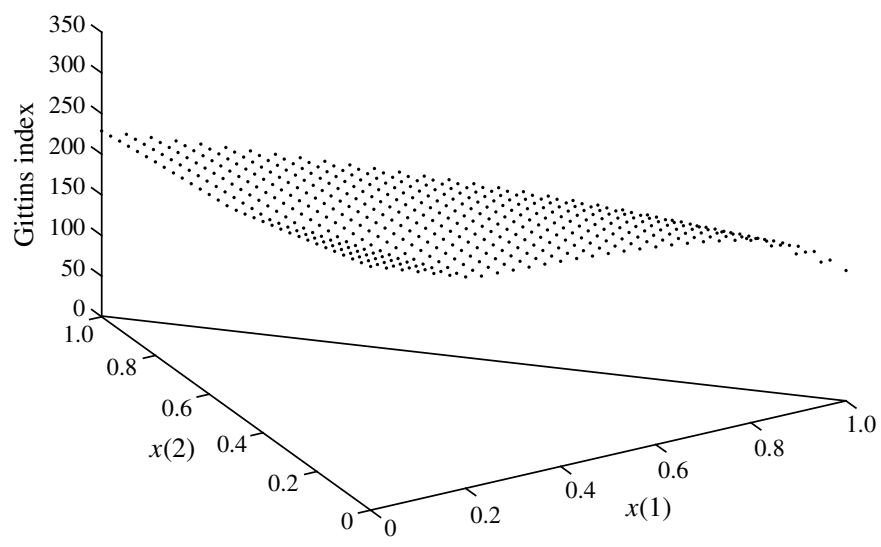

FigURE 2. Approximate Gittins indices computed using Lovejoys's algorithm for $\mathcal{N}=3, \mathcal{M}=3$, and horizon $N=20$.

We simulated the optimal controller using the above computed Gittins index for $P=3$ processes, where each process has the above parameters $A, B, R$. The resulting rewards were compared with that obtained by implementing the linear Gittins index policy (36). The sample path rewards were simulated according to (37) over a horizon of length $N=400$ and averaged more than 200 independent simulations. In each simulation run, we initialized the information states of the three processes as $x_{0}^{(p)}=(1 / 3,1 / 3,1 / 3)$. The rewards obtained using the Gittins index and the linearized index were comparable. As described in $\S 4.2$, setting $\theta(1)=0, \theta(3)=1$, and values of $\theta(2)=0,0.1,0.2, \ldots, 1$ (to ensure that the linear Gittins index is MLR increasing according to Theorem 4.2), the average of the discounted rewards using the linear Gittins index are (see (37)):

225.6540, 225.0375, 225.7653, 224.4294, 228.1006, 223.8780, 225.3717,

$225.0187,225.3916,225.8043,225.6623$

and standard deviations obtained are

$16.6308,18.8187,17.9022,19.5343,18.5426,18.2640,17.4878,17.1489,17.0172,17.8199,17.8673$.

These rewards are similar to the rewards obtained via simulating the policy obtained from the Gittins index computed via Lovejoy's algorithm.

We found that the trajectories of information states generated according to the linear Gittins index policy were MLR orderable quite often. For example, when the information states of the three processes are initialized to $x_{0}^{(p)}=(1 / 3,1 / 3,1 / 3)$, and $\theta(2)=0.8$, the states were not MLR orderable $14.7 \%$ of the time. When the information states are initialized to $x_{0}^{(p)}=e_{3}$ for all three processes, we found that the percentage of times when the $P$ information states were not MLR orderbale was $0.17 \%$, implying that the policy is near optimal. In either case, the discounted rewards obtained are very similar to the above.

As a third example, which also satisfies (A1), (A2), and (A3), we chose

$$
\mathcal{N}=4, \quad M=4, \quad \beta=0.9, \quad A=\left[\begin{array}{cccc}
0.6 & 0.4 & 0.0 & 0.0 \\
0.1 & 0.6 & 0.3 & 0.0 \\
0 & 0.2 & 0.5 & 0.3 \\
0 & 0 & 0.6 & 0.4
\end{array}\right], \quad B=A, \quad R=\left[\begin{array}{c}
9 \\
10 \\
15 \\
20
\end{array}\right]
$$

From Theorem 4.1, $\gamma(x)$ is MLR increasing in $x$. Figure 3 shows the simulated discounted rewards for horizon $N=100$ averaged over 500 independent runs for $P=3$ processes when using the linear approximation to the Gittins index for various values of $\theta(2), \theta(3) \in\{0,0.1,0.2, \ldots, 1\}$ with $\theta(2) \leq \theta(3)$. (As described in $\S 4.2$, $\theta(1)=0, \theta(3)=1$.) Also shown are the standard deviations of the estimated rewards.

In general, we found that the trajectories of information states generated were MLR comparable at most times meaning that the opportunistic policy was optimal. Naturally, the choice of initial state significantly affects the percentage of time that the information states of the $P$ processes are MLR comparable. For five different 


\begin{tabular}{|c|c|c|c|c|c|c|c|c|c|c|c|c|c|}
\hline \multirow[t]{11}{*}{136.7915} & 138.6458 & 138.3323 & \multicolumn{2}{|c|}{138.3626} & \multicolumn{2}{|c|}{137.9544} & 138.6113 & 138.535 & \multicolumn{2}{|c|}{138.2061} & 138.1331 & 138.1121 & 137.9484 \\
\hline & 138.1154 & 138.2894 & \multicolumn{2}{|c|}{138.2400} & \multicolumn{2}{|c|}{138.0982} & 138.3404 & 137.9222 & \multicolumn{2}{|c|}{138.0774} & 137.8110 & 137.8584 & 137.9693 \\
\hline & & 138.1610 & 138.07 & & 138. & 6746 & 138.0251 & 138.262 & \multicolumn{2}{|c|}{138.2643} & 137.9041 & 138.1151 & 137.3271 \\
\hline & & & \multicolumn{2}{|c|}{138.0121} & \multicolumn{2}{|c|}{138.6362} & 138.4387 & 137.610 & \multicolumn{2}{|c|}{137.8523} & 137.7402 & 137.8449 & 137.3559 \\
\hline & & & & & \multicolumn{2}{|c|}{138.4843} & 138.1691 & 137.8848 & \multicolumn{2}{|c|}{137.6662} & 137.6177 & 137.2880 & 137.3084 \\
\hline & & & & & & & 138.1323 & 137.455 & \multicolumn{2}{|c|}{137.3679} & 137.0501 & 137.2254 & 137.3144 \\
\hline & & & & & & & & 136.372 & \multicolumn{2}{|c|}{136.6585} & 136.5739 & 136.4925 & 136.7276 \\
\hline & & & & & & & & & \multicolumn{2}{|c|}{135.1660} & 135.3082 & 135.5659 & 135.7138 \\
\hline & & & & & & & & & & & 135.1906 & 134.9898 & 134.9130 \\
\hline & & & & & & & & & & & & 134.4179 & 133.7014 \\
\hline & & & & & & & & & & & & & 134.2721 \\
\hline & {$[5.4079$} & 4.3573 & 4.5150 & 4.40 & & 4.8772 & 4.0934 & 4.3576 & 4.5637 & 4.476 & $68 \quad 4.2650$ & 4.2722 & 7 \\
\hline & & 4.5897 & 4.5937 & 4.808 & 81 & 4.5008 & 4.2119 & 4.5491 & 4.4074 & 4.529 & $94 \quad 4.2448$ & 4.6061 & \\
\hline & & & 4.5087 & $4.51^{\top}$ & 175 & 4.2000 & 4.7111 & 4.4513 & 4.2721 & 4.326 & $69 \quad 4.1589$ & 4.1385 & \\
\hline & & & & 4.83 & & 4.4112 & 4.2795 & 4.8332 & 4.5457 & 4.44 & $15 \quad 4.2185$ & 4.5693 & \\
\hline & & & & & & 4.4888 & 4.5866 & 4.4534 & 4.5748 & 4.326 & $66 \quad 4.6365$ & 4.8207 & \\
\hline & & & & & & & 4.5591 & 4.4799 & 4.2636 & 4.44 & $49 \quad 4.8086$ & 4.8146 & \\
\hline & & & & & & & & 4.8134 & 4.7341 & 4.713 & $36 \quad 4.4895$ & 4.5571 & \\
\hline & & & & & & & & & 5.6716 & 5.22 & $40 \quad 5.5015$ & 5.3147 & \\
\hline & & & & & & & & & & 5.60 & $08 \quad 5.7457$ & 5.7909 & \\
\hline & & & & & & & & & & & 5.8836 & 6.3588 & \\
\hline & L & & & & & & & & & & & 6.2194 & \\
\hline
\end{tabular}

Figure 3. Simulated discounted reward (37) of linear Gittins index policy (36) as a function of linear coefficients $\theta(2), \theta(3) \in$ $\{0,0.1,0.2, \ldots, 1\}$ with $\theta(2) \leq \theta(3)$. Equation (44) shows the average of the discounted reward over 500 independent simulation runs and (45) shows the standard deviation.

initializations depicted in the table below, we show the percentages of time $k$ the states $x_{k}^{(p)}, p=1,2,3$, are not MLR comparable for $\theta=\left[\begin{array}{llll}0,0.5, & 0.8,1\end{array}\right]^{\prime}$.

\begin{tabular}{lccccc}
\hline$x_{0}^{(p)}$ & $e_{1}$ & $e_{2}$ & $e_{3}$ & $e_{4}$ & $x_{0}^{(p)}(i)=1 / 4$ \\
\hline Percent of time states not MLR orderable & 0 & 10.5 & 9.82 & 3.44 & 36.4 \\
\hline
\end{tabular}

In general, we found that the more diagonally dominant $A$ and $B$ were, the less the percentage of cases where the states were not MLR orderable.

We also ran the stochastic approximation algorithm of $\S 4.2$ to estimate the coefficients of the best linear fit to the Gittins index. For the examples presented above, the choice of $\theta$ has little effect on the sample path reward. For examples with $\mathcal{N}=10, M=10$, optimizing $\theta$ did improve the reward of the policy-particularly when the trajectories were not MLR orderable.

REMARK 5.1. For the three examples presented in the discussion of $\S 4.1$ (namely, (i) $\mathcal{N}=2$ and arbitrary $\mathscr{M}$, (ii) bidiagonal $B$ and arbitrary $\mathcal{N}$, and (iii) uniform $B$ ), if (A1), (A2), and (A3) hold, then the optimal policy is opportunistic by Theorem 4.1. So we did not present numerical examples for these cases as they are trivial to implement. In addition, we found that for several diagonally dominant tridiagonal $A$ and $B$ matrices, the information state trajectories were MLR comparable at all time points. (However, we are unable to prove this.)

REMARK 5.2. Numerical experiments not presented here show that for problems with processes having up to $\mathcal{N}=5$ states, the incremental prune algorithm and Lovejoy's lower-bound algorithm can be satisfactorily used. The $\mathrm{C}++$ code for implementing the POMDP value iteration algorithm was downloaded from Cassandra [2]. The Matlab code for computing the Gittins indices and implementing Lovejoy's algorithm are freely available from the author at vikramk@ece.ubc.ca. The SPSA algorithm was implemented in Matlab. 
6. Conclusion. We have presented a value iteration-based algorithm for providing a finite-dimensional characterization of the Gittins index of a POMDP multiarmed bandit problem. We also presented structural results where the Gittins index is monotone increasing in the information state with respect to the MLR order. In such cases, the optimal policy is opportunistic in the information state (when the states are MLR comparable). When they are not MLR comparable, we presented a linear approximation to the Gittins index that is MLR increasing.

In related work we have derived structural results for POMDPs in radar/sensor scheduling in Krishnamurthy and Djonin [7]

Acknowledgments. This research was supported by NSERC, Canada; the Swedish Science Foundation; and the Linnaeus Center ACCESS at KTH.

\section{References}

[1] Bertsimas, D., J. Nino-Mora. 1996. Conservation laws, extended polymatroids and multiarmed bandit problems; a polyhedral approach to indexable systems. Math. Oper. Res. 21(2) 257-305.

[2] Cassandra, A. R. Tony's POMDP page. http://www.cs.brown.edu/research/ai/pomdp/index.html.

[3] Cassandra, A. R. 1998. Exact and approximate algorithms for partially observed Markov decision process. Doctoral dissertation, Brown University, Providence, RI.

[4] Cassandra, A. R., M. L. Littman, N. L. Zhang. 1997. Incremental pruning: A simple fast exact method for partially observed Markov decision processes. Proc. 13th Annual Conf. Uncertainty in Artificial Intelligence (UAI-97). Morgan Kaufmann, San Francisco.

[5] Gittins, J. C. 1989. Multi-Armed Bandit Allocation Indices. John Wiley and Sons, New York.

[6] Kijima, M. 1997. Markov Processes for Stochastic Modelling. Chapman and Hall, London.

[7] Krishnamurthy, V., D. Djonin. 2007. Structured threshold policies for dynamic sensor scheduling-A partially observed Markov decision process approach. IEEE Trans. Signal Processing 55(10) 4938-4957.

[8] Krishnamurthy, V., F. J. Vázquez-Abad, K. Martin. 2003. Implementation of gradient estimation to a constrained Markov decision problem. 42nd IEEE Conf. Decision and Control, Maui, Hawaii. IEEE Press, Piscataway, NJ.

[9] Kumar, P. R., P. Varaiya. 1986. Stochastic Systems-Estimation, Identification and Adaptive Control. Prentice-Hall, Upper Saddle River, NJ.

[10] Kushner, H. J., G. Yin. 1987. Stochastic approximation algorithms for parallel and distributed processing. Stochastics 22 219-250.

[11] Le Cadre, J. P., O. Trémois. 1998. Bearings-only tracking for maneuvering sources. IEEE Trans. Aerospace Electronic Systems 34(1) $179-193$.

[12] Lovejoy, W. S. 1987. Some monotonicity results for partially observed Markov decision processes. Oper. Res. 35(5) $736-743$.

[13] Lovejoy, W. S. 1991. Computationally feasible bounds for partially observed Markov decision processes. Oper. Res. 39(1) 162-175.

[14] Muller, A., D. Stoyan. 2002. Comparison Methods for Stochastic Models and Risk. John Wiley \& Sons, Chichester, UK.

[15] Papadimitriou, C. H. 1995. Computational Complexity. Addison-Wesley, Reading, MA.

[16] Rabiner, L. R. 1989. A tutorial on hidden Markov models and selected applications in speech recognition. Proc. IEEE 77(2) 257-285.

[17] Ross, S. 1983. Introduction to Stochastic Dynamic Programming. Academic Press, San Diego.

[18] Smallwood, R. D., E. J. Sondik. 1973. Optimal control of partially observable Markov processes over a finite horizon. Oper. Res. 21 1071-1088.

[19] Spall, J. 2003. Introduction to Stochastic Search and Optimization. John Wiley and Sons, New York.

[20] Whittle, P. 1980. Multi-armed bandits and the Gittins index. J. R. Statist. Soc. B 42(2) 143-149. 